\title{
UM PROBLEMA DE CORTE COM PADRÕES COMPARTIMENTADOS
}

\author{
Robinson Hoto * \\ CCE - Departamento de Matemática \\ Universidade Estadual de Londrina \\ Londrina - PR \\ hoto@uel.br
}

Nelson Maculan

COPPE - Prog. de Eng. de Sistemas e Computação

Universidade Federal do Rio de Janeiro

Rio de Janeiro - RJ

maculan@cos.ufrj.br

Fabiano Marques

Marcos Arenales

ICMC - Departamento de Computação e Estatística

Universidade de São Paulo

São Carlos - SP

araxa@icmc.sc.usp.br; arenales@icmc.sc.usp.br

* Corresponding author/autor para quem as correspondências devem ser encaminhadas

Recebido em 06/2002, aceito em 11/2002 após 1 revisão

\section{Resumo}

Neste artigo apresentaremos a aplicação do Problema da Mochila Compartimentada (PMC) no Problema de Corte de Bobinas de Aço (PCBA), que é um problema de corte em duas etapas com restrições especiais de agrupamento dos itens. O PMC consiste em construir compartimentos de capacidades desconhecidas em uma mochila de capacidade conhecida, tendo em vista que os itens de interesse estão agrupados em subconjuntos, de modo que, itens de um agrupamento não podem ser combinados com itens de outro. Para entender melhor o PMC admita que a mochila de um alpinista deve ser composta por um número ideal de compartimentos com itens de quatro categorias (remédios, alimentos, ferramentas, roupas), porém, itens de categorias distintas não podem ser combinados para formar um mesmo compartimento, além do mais, são desconhecidas as capacidades ideais de cada compartimento da mochila.

Palavras-chave: corte e empacotamento; padrões compartimentados; mochila compartimentada.

\begin{abstract}
In this paper we will present the application of the Compartmented Knapsack Problem (CKP) in the Cut Problem of Steel Rolls (CPSR), that it is a problem of cut in two stages with restrictions special of grouping of items. The CKP consists of constructing compartments of unknown capacities in a knapsack of known capacity, in view of that items of interest is grouped in subgroups, in mode that, items of a grouping cannot be matched with items of another one. To understand the CKP more good it admits that the knapsack of a alpinist must be composite for an ideal number of compartments with items of four categories (remedies, foods, tools, clothes), however, items of distinct categories cannot be matched to form one same compartment, in addition, is unknown the ideal capacities of each compartment of the knapsack.
\end{abstract}

Keywords: cutting and packing problem; compartmented pattern; compartmented knapsack. 


\section{Introdução}

Padrões de corte compartimentados são comuns em empresas do ramo da metalurgia que necessitam efetuar laminação a frio do aço que é acondicionado em bobinas. Estas bobinas do estoque são identificadas pelo seu "peso" (varia de $12.000 \mathrm{Kg}$ a $13.500 \mathrm{Kg}$ ), sua largura (varia de $1000 \mathrm{~mm}$ a $1300 \mathrm{~mm}$ ), espessura do aço (varia de $0,90 \mathrm{~mm}$ a $6,30 \mathrm{~mm}$ ) e pelo teor de carbono na liga, que determinará a dureza do aço. A demanda é composta por fitas de especificações determinadas (largura, espessura e tipo de aço) que devem ser cortadas de uma bobina do estoque e, devido a razões técnicas, a maioria destas fitas precisam ter a espessura do aço reduzida por meio de laminação. A laminação é feita com "cilindros de laminação" que efetuam pressão sobre a lâmina de aço em temperatura ambiente (laminação a frio, figura 1). Devido a limitações técnicas, a máquina de laminação não pode processar a largura total de uma bobina do estoque, além do mais, existem vários conjuntos de fitas com reduções de espessura de aço distintas, de modo que, devem ser definidas bobinas intermediárias com larguras admissíveis pelo laminador e compostas de fitas compatíveis entre si. Estas bobinas intermediárias são cortadas de uma bobina em estoque e, delas são recortadas as fitas, de modo que, os padrões de corte devem ser estruturados em compartimentos, cujos tamanhos devem ser determinados.

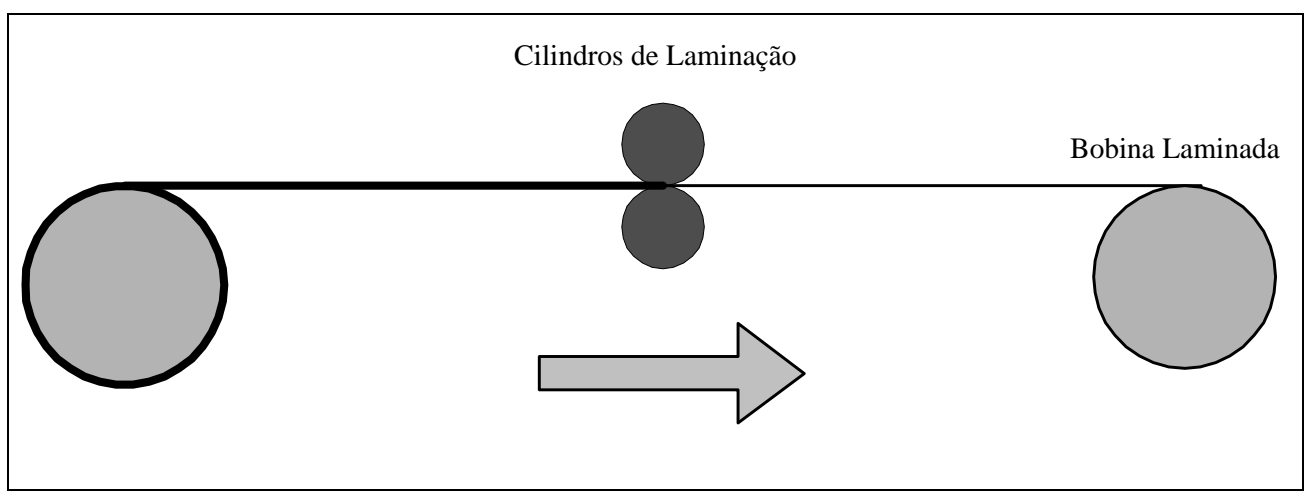

Figura 1 - Laminação a frio do aço de uma bobina

Na figura 2 ilustramos o processo de corte com um padrão compartimentado. Suponha que o aço da bobina tenha $1,20 \mathrm{~mm}$ de espessura e que as fitas da primeira bobina intermediária precisem ser laminadas a uma espessura de $1,00 \mathrm{~mm}$, enquanto, as da segunda bobina intermediária devem ser laminadas a uma espessura de $0,90 \mathrm{~mm}$, ou numa outra situação não serem laminadas. 


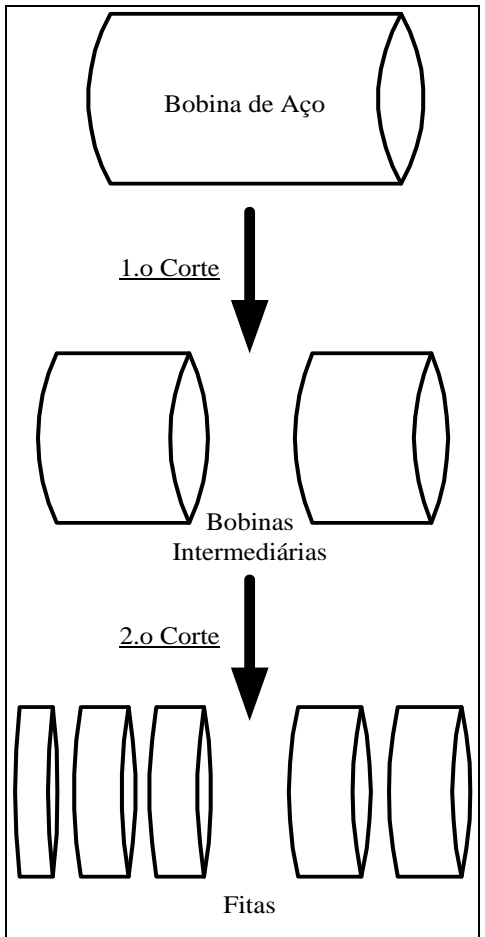

Figura 2 - Primeira e segunda fases de corte com um padrão compartimentado

Por razões técnicas, o número de cortes numa bobina do estoque e de recortes numa bobina intermediária é limitado, além do mais, nas bobinas do estoque e nas bobinas intermediárias existem perdas técnicas intrínsecas.

Em síntese, os aspectos peculiares aqui citados apontam para um problema complexo e que foge do contexto clássico, até porque, o esquema de corte se dá em duas fases. Além disto, a necessidade do processo de laminação induz padrões de corte nada evidentes, visto que, nem todas as fitas são compatíveis entre si.

\section{Padrões de Corte Compartimentados}

Um padrão de corte é denominado compartimentado quando é necessário definir compartimentos (objetos intermediários) a serem cortados dos objetos, dos quais posteriormente serão recortados os itens. Este tipo de padrão ocorre quando os itens necessitam sofrer processos técnicos distintos, ou máquinas da linha de produção não comportam as dimensões dos objetos em estoque.

Um exemplo é o da indústria de móveis, onde os itens devem passar por processos técnicos distintos e as máquinas da linha de produção não comportam as dimensões originais das placas de madeira. Assim, há a necessidade de construir padrões compartimentos (figura 3), onde cada um deles corresponde a uma placa intermediária de dimensões menores que a da placa original. 


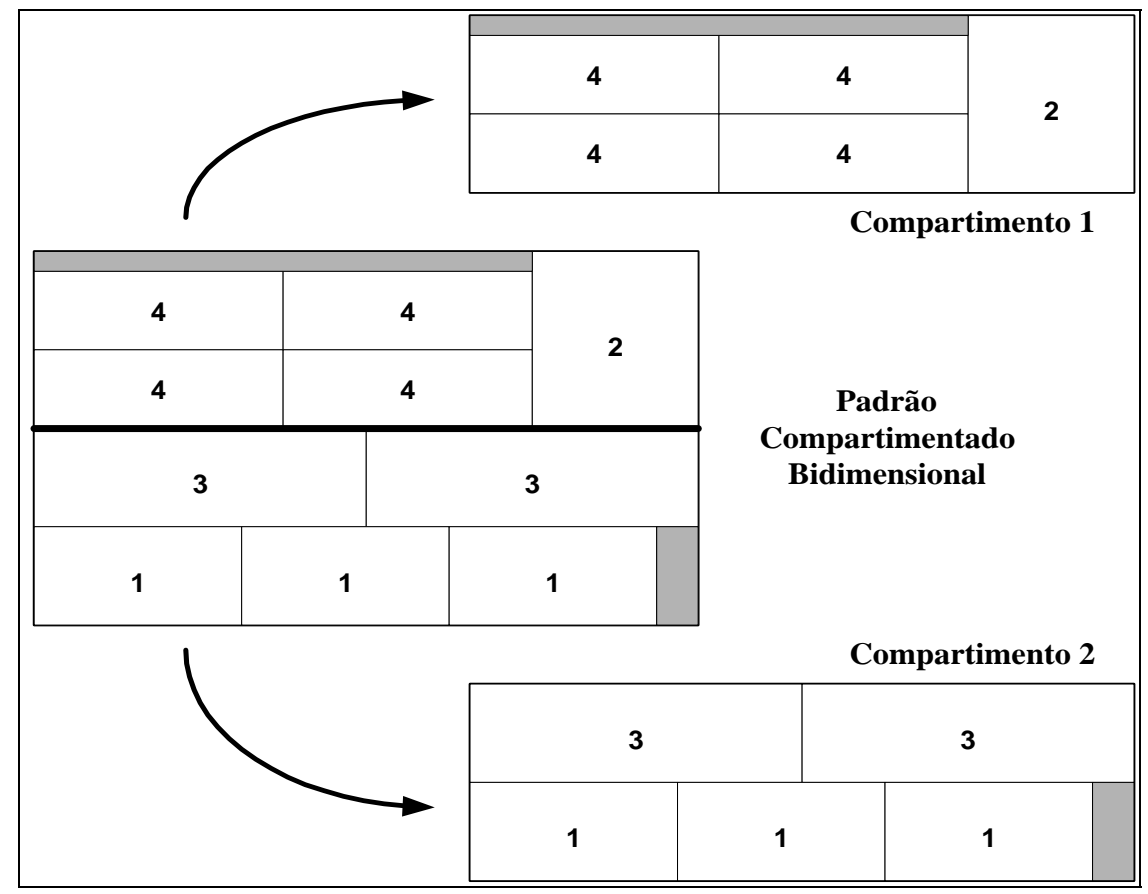

Figura 3 - Padrão de corte compartimentado bidimensional

O corte de bobinas de aço é um exemplo onde aparecem padrões compartimentados unidimensionais, pois, alguns itens devem sofrer laminações distintas, neste caso é preciso definir compartimentos que são denominados bobinas intermediárias (figura 2).

A grande dificuldade na construção de um padrão compartimentado, unidimensional ou bibdimensional, está no fato de não conhecermos as dimensões dos compartimentos que devem compor o padrão, ao mesmo tempo, é preciso determinar quais compartimentos e em quais quantidades constituirão o padrão. Hoto (2001) e Hoto et al. (1996, 1996, 1998) descrevem padrões compartimentados unidimensionais por meio de um Problema da Mochila Compartimentada (PMC) (Hoto et al., 1999, 2002), que é uma nova variação do clássico Problema da Mochila. Marques (2000) e Marques \& Arenales (2000) estudaram o caso restrito do PMC e apresentaram alguns procedimentos heurísticos. Na próxima seção apresentaremos o modelo de geração de colunas que escrevemos para o corte de bobinas de aço, bem como uma formulação matemática da mochila compartimentada para gerar padrões compartimentados unidimensionais.

\section{Uma abordagem de Geração de Colunas no Corte de Bobinas de Aço}

Na resolução do Problema de Corte de Bobinas de Aço (PCBA) sujeitas a laminação adotamos a Técnica de Geração de Colunas de Gilmore-Gomory (1961, 1963). Nosso objetivo consiste em minimizar os custos associados às perdas lineares de aço numa bobina, e custos por utilização de compartimentos, além de examinar as limitações do estoque. Considere as definiçõos dadas a seguir: 


\section{Dados das Bobinas}

- $\mathrm{m}$ total de tipos de bobinas em estoque;

- $\mathrm{P}^{\mathrm{r}} \quad$ "peso" (a massa) da bobina (objeto) $\mathrm{r}=1, \ldots, \mathrm{m}(\mathrm{Kg})$;

- $\mathrm{L}^{\mathrm{r}} \quad$ largura da bobina (objeto) $\mathrm{r}=1, \ldots, \mathrm{m}(\mathrm{mm})$;

- $\mathrm{e}^{\mathrm{r}} \quad$ disponibilidade da bobina (objeto) $\mathrm{r}=1, \ldots, \mathrm{m}$ (unidades de bobinas);

- $\mathrm{c}_{\text {aço }}^{\mathrm{r}} \quad$ custo linear do aço da bobina (objeto) $\mathrm{r}=1, \ldots, \mathrm{m}(\$ / \mathrm{mm})$;

- $\mathrm{T}_{\mathrm{j}}^{\mathrm{r}}$ perda linear de aço na bobina (objeto) $\mathrm{r}=1, \ldots, \mathrm{m}$, segundo o padrão compartimentado j (mm);

- $\mathrm{S}_{1} \quad$ perda intrínseca na borda de uma bobina $(\mathrm{mm})$;

\section{Dados das Fitas}

- $\mathrm{n}$ total de tipos de itens;

- $\ell_{\mathrm{i}} \quad$ largura da fita (item) $\mathrm{i}=1, \ldots, \mathrm{n}(\mathrm{mm})$;

- $\mathrm{d}_{\mathrm{i}}$ demanda solicitada da fita (item) $\mathrm{i}=1, \ldots, \mathrm{n}(\mathrm{Kg})$;

- $\mathrm{a}_{\mathrm{i} j}^{\mathrm{r}} \quad$ número de fitas (itens) tipo $\mathrm{i}$ na bobina $\mathrm{r}$, segundo o padrão compartimentado $\mathrm{j}$ (unidades de fitas);

\section{Dados das Bobinas Intermediárias}

- $\quad \mathrm{c}_{\mathrm{hj}}^{\mathrm{r}} \quad$ custo operacional da bobina intermediária (compartimento) h na bobina $\mathrm{r}$, segundo o padrão compartimentado j (\$/unidade do compartimento $h$ );

- $\mathrm{H}_{\mathrm{jr}}$ total de tipos de bobinas intermediárias (compartimentos) na bobina $\mathrm{r}$, segundo o padrão compartimentado $\mathrm{j}$ (unidades de compartimentos);

- $y_{\mathrm{hj}}^{\mathrm{r}}$ número de bobinas intermediárias (compartimentos) h na bobina $\mathrm{r}$, segundo o padrão compartimentado j (unidades de compartimentos);

- $\mathrm{L}_{\min }$ largura mínima de uma bobina intermediária (mm);

- $\mathrm{L}_{\max }$ largura máxima de uma bobina intermediária $(\mathrm{mm})$;

- $\mathrm{S}_{2}$ perda intrínseca na borda de uma bobina intermediária $(\mathrm{mm})$;

\section{Padrões}

- $\mathrm{p}_{\mathrm{r}} \quad$ total de padrões compartimentados viáveis para bobinas do tipo r;

\section{Variável}

- $\mathrm{X}_{\mathrm{j}}^{\mathrm{r}}$ número de bobinas utilizadas do tipo $\mathrm{r}$, segundo o padrão compartimentado $\mathrm{j}$ (unidades de bobinas); 
O modelo matemático que formulamos para resolver o PCBA é escrito como:

\section{Modelo do PCBA}

$\operatorname{minimizar} \sum_{r=1}^{m} \sum_{j=1}^{p_{r}}\left(c_{a c ̧ o ~}^{r} T_{j}^{r}+\sum_{h=1}^{H_{j r}}\left(c_{h j}^{r} y_{h j}^{r}\right)\right) x_{j}^{r}$

sujeito a:

$$
\begin{array}{cc}
\sum_{r=1}^{m} \sum_{j=1}^{p_{r}} \frac{P^{r}}{L^{r}} \ell_{i} a_{i j}^{r} x_{j}^{r}= & d_{i}, i=1, \ldots, n \\
\sum_{j=1}^{p_{1}} x_{j}^{1} & \leq e^{1} \\
\ddots & \vdots \\
x_{j}^{r} \geq 0, \text { e inteiro } \quad r=1, \ldots, m, j=1, \ldots, p_{r}
\end{array}
$$

Para facilitar cálculos e notações, sejam $c_{j}^{r}=c_{a c ̧ o}^{r} T_{j}^{r}+\sum_{h=1}^{H_{j r}}\left(c_{h j}^{r} y_{h j}^{r}\right)$ e $\alpha_{i j}^{r}=\frac{P^{r}}{L^{r}} \ell_{i} a_{i j}^{r}$. Na figura 4 ilustramos a estrutura matricial do modelo, onde está representada a matriz A e os vetores c e d, e onde $A_{r}=\left(\alpha_{i j}^{r}\right)_{n \times p_{r}}, r=1, \ldots, m$.

Observe que uma coluna do modelo do PCBA $(1.1-1.4)$ possui $(n+m)$ componentes e tem o seguinte aspecto $\alpha_{j}^{r}=\left(\alpha_{1 j}^{r}, \alpha_{2 j}^{r}, \ldots, \alpha_{n j}^{r}, 0, \ldots, 0,1,0, \ldots, 0\right)^{T}$, onde o valor unitário ocupa a posição $(n+r)$ da coluna.

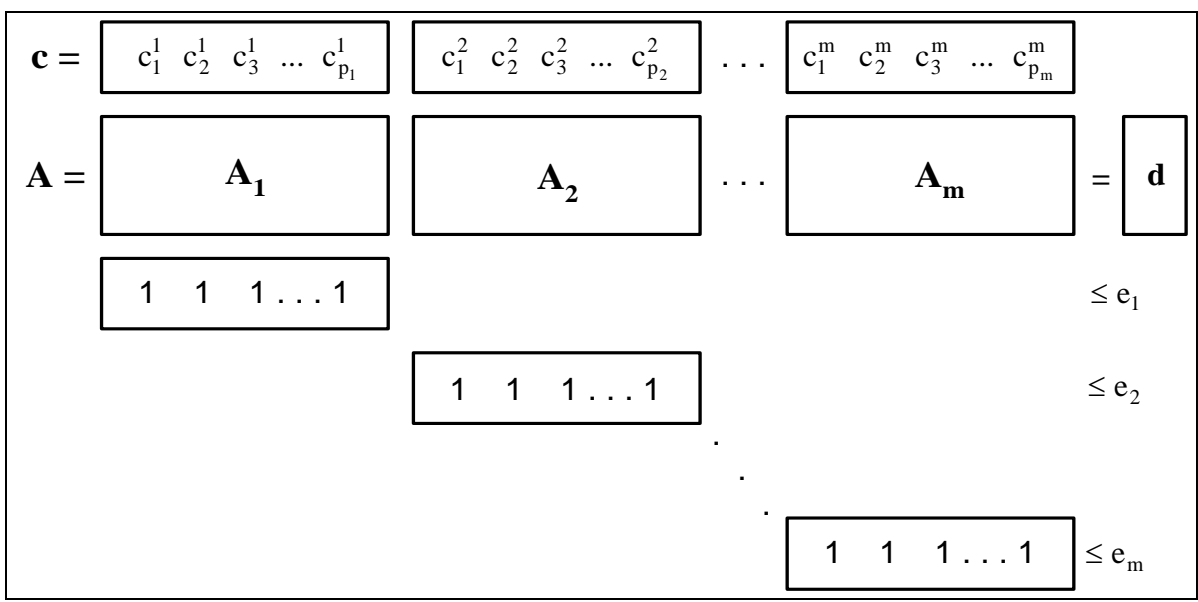

Figura 4 - Estrutura matricial do modelo do PCBA (sem o vetor x de variáveis) 
Devido a grandeza da matriz A, na prática ela pode atingir milhões ou bilhões de colunas, a utilização do SIMPLEX fica prejudicada, pois, decidir qual coluna deverá compor a nova base dentre as milhões ou bilhões existentes é computacionalmente inviável. Para contornar este entrave, Gilmore-Gomory $(1961,1963)$ introduziram a idéia de determinar esta coluna (no caso do PCBA uma coluna do tipo $\alpha_{j}^{r}$ ) por meio de um problema otimização que depende das características das colunas. Para escrevermos este problema, iremos investigar a natureza dos custos relativos para cada tipo de bobina $\mathrm{r}=1, \ldots, \mathrm{m}$ do estoque. Seja o vetor de multiplicadores SIMPLEX dado por $\pi=\mathrm{c}_{\mathrm{B}} \mathrm{B}^{-1}=\left(\pi_{1}, \ldots, \pi_{\mathrm{n}}, \pi_{\mathrm{n}+1}, \ldots, \pi_{\mathrm{n}+\mathrm{r}}, \ldots, \pi_{\mathrm{n}+\mathrm{m}}\right)$, onde $\mathrm{B}$ é uma matriz básica obtida de $\mathrm{A}$ e $\mathrm{c}_{\mathrm{B}}$ o vetor de custos associado às colunas de $\mathrm{B}$, então:

$$
\begin{gathered}
c_{j}^{r}-\pi \alpha_{j}^{r}=c_{j}^{r}-\left(\sum_{i=1}^{n} \pi_{i} \alpha_{i j}^{r}+\pi_{n+r}\right)= \\
=c_{a c ̧ o ~}^{r} T_{j}^{r}+\sum_{h=1}^{H_{j r}}\left(c_{h j}^{r} y_{h j}^{r}\right)-\left(\sum_{i=1}^{n} \pi_{i} \alpha_{i j}^{r}+\pi_{n+r}\right)= \\
=c_{a c ̧ o}^{r}\left(\left(L^{r}-S_{1}\right)-\left(\sum_{i=1}^{n} \ell_{i} a_{i j}^{r}\right)-\left(S_{2} \sum_{h=1}^{H_{j r}} y_{h j}^{r}\right)\right)+\left(\sum_{h=1}^{H_{j r}} c_{h j}^{r} y_{h j}^{r}\right)-\left(\sum_{i=1}^{n} \pi_{i} \alpha_{i j}^{r}+\pi_{n+r}\right)
\end{gathered}
$$

Substituindo $\alpha_{i j}^{r}$ por $\frac{P^{r}}{L^{r}} \ell_{i} a_{i j}^{r}$ e reorganizando o cálculo obtemos:

$$
\begin{gathered}
c_{j}^{r}-\pi \alpha_{j}^{r}= \\
=-\sum_{i=1}^{n}\left(c_{a c ̧ o}^{r}+\pi_{i} \frac{P^{r}}{L^{r}}\right) \ell_{i} a_{i j}^{r}+\sum_{h=1}^{H_{j r}}\left(c_{h j}^{r}-c_{a c ̧ o}^{r} S_{2}\right) y_{h j}^{r}+\left(c_{a c ̧ o}^{r}\left(L^{r}-S_{1}\right)-\pi_{n+r}\right)
\end{gathered}
$$

Escrevendo $a_{i j}^{r}=\sum_{h=1}^{H_{j r}} a_{i h}^{j r} y_{h j}^{r}$, onde $a_{i h}^{j r}$ é o número de itens tipo $i$ que aparecem no compartimento h, do padrão j que é usado para cortar bobinas do tipo r, a expressão do custo relativo pode ser rescrita como:

$$
\begin{gathered}
c_{j}^{r}-\pi \alpha_{j}^{r}= \\
=-\left(\sum_{i=1}^{n}\left(c_{a c ̧ o}^{r}+\pi_{i} \frac{P^{r}}{L^{r}}\right) \ell_{i} \sum_{h=1}^{H_{j r}} a_{i h}^{j r} y_{h j}^{r}\right)+\left(\sum_{h=1}^{H_{j r}}\left(c_{h j}^{r}-c_{a c ̧}^{r} S_{2}\right) y_{h j}^{r}\right)+\left(c_{a c ̧ o}^{r}\left(L^{r}-S_{1}\right)-\pi_{n+r}\right)
\end{gathered}
$$

Finalmente a expressão do custo relativo é escrita da seguinte forma:

$$
c_{j}^{r}-\pi \alpha_{j}^{r}=-\left(\sum_{h=1}^{H_{j r}}\left(\left(\sum_{i=1}^{n} u_{i} a_{i h}^{j r}\right)-c_{h}\right) y_{h j}^{r}\right)+\left(c_{a c ̧ o}^{r}\left(L^{r}-S_{1}\right)-\pi_{n+r}\right)
$$

Onde,

$$
u_{i}=\left(c_{\text {aço }}^{r}+\pi_{i} \frac{P^{r}}{L^{r}}\right) \ell_{i} \text { e } \quad c_{h}=c_{h j}^{r}-c_{a c ̧ o}^{r} S_{2}
$$


Note que para cada bobina do tipo $r$, a parcela $c_{\text {aço }}^{r}\left(L^{r}-S_{1}\right)-\pi_{n+r}$ de (2.1) é uma constante, além do mais, os itens do PCBA (as fitas) podem ser indexados pelo conjunto $\mathrm{N}=\{1, \ldots, \mathrm{n}\}$, e eles devem ser agrupados em subconjuntos $\mathrm{N}_{1}, \ldots, \mathrm{N}_{\mathrm{k}}$, onde cada um deles contém os índices das fitas que são compatíveis entre si devido o processo de laminação, de forma que $\left\{\mathrm{N}_{1}, \ldots, \mathrm{N}_{\mathrm{k}}\right\}$ constitua uma partição de $\mathrm{N}$.

Para cada subconjunto $\mathrm{N}_{\mathrm{s}}, \mathrm{s}=1, \ldots, \mathrm{k}$, seja o conjunto $\mathrm{V}_{\mathrm{s}}$ dos índices dos compartimentos viáveis construídos a partir dos itens de $\mathrm{N}_{\mathrm{s}}$, de forma que $\left\{\mathrm{V}_{1}, \ldots, \mathrm{V}_{\mathrm{k}}\right\}$ constitua uma partição de $\mathrm{V}=\mathrm{V}_{1} \cup \cdots \cup \mathrm{V}_{\mathrm{k}}$. Tendo em vista que devemos obter o mínimo $\left\{\mathrm{c}_{\mathrm{j}}^{\mathrm{r}} \mathrm{T}_{\mathrm{j}}^{\mathrm{r}}-\pi \alpha_{\mathrm{j}}^{\mathrm{r}} \mid \alpha_{\mathrm{j}}^{\mathrm{r}}\right.$ é uma coluna não básica $\}$, o problema gerador de colunas do modelo $(1.1-1.4)$ pode ser escrito como:

\section{Modelo do Gerador de Padrões Compartimentados do PCBA}

$$
\operatorname{maximizar} \sum_{h \in V_{1}}\left(\left(\sum_{i \in N_{1}} u_{i} a_{i h}\right)-c_{h}\right) y_{h}+\cdots+\sum_{h \in V_{k}}\left(\left(\sum_{i \in N_{k}} u_{i} a_{i h}\right)-c_{h}\right) y_{h}
$$

sujeito a:

$$
\begin{aligned}
& \sum_{\mathrm{h} \in \mathrm{V}_{1}}\left(\mathrm{~S}_{2}+\sum_{\mathrm{i} \in \mathrm{N}_{\mathrm{l}}} \ell_{\mathrm{i}} \mathrm{a}_{\mathrm{ih}}\right) \mathrm{y}_{\mathrm{h}}+\cdots+\sum_{\mathrm{h} \in \mathrm{V}_{\mathrm{k}}}\left(\mathrm{S}_{2}+\sum_{\mathrm{i} \in \mathrm{N}_{\mathrm{k}}} \ell_{\mathrm{i}} \mathrm{a}_{\mathrm{ih}}\right) \mathrm{y}_{\mathrm{h}} \leq \mathrm{L}-\mathrm{S}_{1} \\
& \mathrm{~L}_{\text {min }}-\mathrm{S}_{2} \leq \sum_{\mathrm{i} \in \mathrm{N}_{\mathrm{s}}} \ell_{\mathrm{i}} \mathrm{a}_{\mathrm{ih}} \leq \mathrm{L}_{\max }-\mathrm{S}_{2}, \mathrm{~h} \in \mathrm{V}_{\mathrm{s}}, \mathrm{s}=1, \ldots, \mathrm{k} \\
& \mathrm{a}_{\mathrm{ih}}, \mathrm{y}_{\mathrm{h}} \geq 0 \text { e inteiros, } \\
& \mathrm{i} \in \mathrm{N}=\mathrm{N}_{1} \cup \cdots \cup \mathrm{N}_{\mathrm{k}}, \mathrm{h} \in \mathrm{V}=\mathrm{V}_{1} \cup \cdots \cup \mathrm{V}_{\mathrm{k}}
\end{aligned}
$$

O modelo (3.1 - 3.4) descreve o que denominamos Problema da Mochila Compartimentada (PMC). Observe que (3.2) é a restrição física da mochila e (3.3) são chamadas de "restrições de compartimentação". O PMC pode ser resolvido otimamente pelo seguinte procedimento:

\section{Procedimento COMPEX}

1. Para $\mathrm{s}=1, \ldots, \mathrm{k}$, seja o agrupamento $\mathrm{N}_{\mathrm{s}}$ e o subconjunto $\mathrm{V}_{\mathrm{s}}$ associado;

1.1 Para cada $\mathrm{h} \in \mathrm{V}_{\mathrm{s}}$, considere $\mathrm{w}_{\mathrm{h}} \in\left\{\left(\mathrm{L}_{\min }-\mathrm{S}_{2}\right),\left(\mathrm{L}_{\min }-\mathrm{S}_{2}\right)+1, \ldots,\left(\mathrm{L}_{\max }-\mathrm{S}_{2}\right)\right\}$ a capacidade do compartimento viável de índice $\mathrm{h}$, e resolva a seguinte mochila para obter a utilidade $\mathrm{V}_{\mathrm{h}}$ do compartimento:

$$
\operatorname{maximizar} \mathrm{v}_{\mathrm{h}}=\sum_{\mathrm{i} \in \mathrm{N}_{\mathrm{s}}} \mathrm{u}_{\mathrm{i}} \mathrm{a}_{\mathrm{ih}}
$$

sujeito a:

$$
\begin{aligned}
& \sum_{\mathrm{i} \in \mathrm{N}_{\mathrm{s}}} \ell_{\mathrm{i}} \mathrm{a}_{\mathrm{ih}}=\mathrm{w}_{\mathrm{h}} \\
& \mathrm{a}_{\mathrm{ih}} \geq 0 \text { e inteiro, } \mathrm{i} \in \mathrm{N}_{\mathrm{s}}
\end{aligned}
$$


2. Seja $V=V_{1} \cup \cdots \cup V_{k}$ e resolva a seguinte mochila:

$$
\operatorname{maximizar} \sum_{\mathrm{h} \in \mathrm{V}}\left(\mathrm{v}_{\mathrm{h}}-\mathrm{c}_{\mathrm{h}}\right) \mathrm{y}_{\mathrm{h}}
$$

sujeito a:

$$
\begin{aligned}
& \sum_{\mathrm{h} \in \mathrm{V}} \mathrm{w}_{\mathrm{h}} \mathrm{y}_{\mathrm{h}} \leq \mathrm{L}-\mathrm{S}_{1} \\
& \mathrm{y}_{\mathrm{h}} \geq 0 \text { e inteiro, } \mathrm{h} \in \mathrm{V}
\end{aligned}
$$

\section{Propriedade}

O algoritmo COMPEX encontra uma solução ótima do Problema da Mochila Compartimentada (PMC), quando todas as mochilas do procedimento forem resolvidas otimamente.

Prova Para cada compartimento de índice $\mathrm{h} \in \mathrm{V}$, existe um único $\mathrm{s} \in\{1, \ldots, \mathrm{k}\}$ que depende de $h$, tal que $h \in V_{s}$, visto que os conjuntos $V_{s}, s=1, \ldots, k$, foram construídos como uma partição de $\mathrm{V}$.

Seja $\mathrm{w}_{\mathrm{h}}$ a capacidade deste compartimento e considere $\Omega_{\mathrm{h}}=\left\{\left(\mathrm{a}_{\mathrm{ih}}\right)_{\mathrm{i} \in \mathrm{N}_{\mathrm{s}}} \mid \mathrm{w}_{\mathrm{h}}=\sum_{\mathrm{i} \in \mathrm{N}_{\mathrm{s}}} \mathrm{p}_{\mathrm{i}} \mathrm{a}_{\mathrm{ih}}\right\} \mathrm{o}$ conjunto de todos os vetores que representam uma combinação linear dos pesos dos itens com índices em $\mathrm{N}_{\mathrm{s}}$, igual a $\mathrm{w}_{\mathrm{h}}$.

A cardinalidade de $\Omega_{\mathrm{h}}$ é finita, assim suponha que $\Omega_{\mathrm{h}}$ tenha $\mathrm{m}_{\mathrm{h}}$ vetores e considere $\mathrm{v}_{\mathrm{t}}^{\mathrm{h}}=\sum_{\mathrm{i} \in \mathrm{N}_{\mathrm{s}}} \mathrm{u}_{\mathrm{i}} \mathrm{a}_{\mathrm{ih}}, \mathrm{t}=1, \ldots, \mathrm{m}_{\mathrm{h}}$ os possíveis valores de utilidades para o compartimento de capacidade $\mathrm{w}_{\mathrm{h}}$.

Observe que ao escolhermos $\mathrm{v}_{\mathrm{h}}=\max \left\{\mathrm{v}_{1}^{\mathrm{h}}, \ldots, \mathrm{v}_{\mathrm{m}_{\mathrm{h}}}^{\mathrm{h}}\right\}$ (mochila do passo 1 do COMPEX) a utilidade $\mathrm{v}_{\mathrm{h}}$ é dominante sob todas as possíveis utilidades do compartimento de capacidade $\mathrm{w}_{\mathrm{h}}$, e este mesmo raciocínio se aplica ao custo $\mathrm{c}_{\mathrm{h}}$ por utilização do compartimento.

Supondo que a mochila do passo 2 do COMPEX é resolvida otimamente podemos concluir que o COMPEX encontra um ótimo para o PMC.

Um procedimento alternativo, baseado no cálculo de limitantes, pode ser usado para obter uma solução viável do PMC. Neste caso, a alteração é feita no passo 1.1 do COMPEX, vejamos: 


\section{Procedimento COMPMT}

1. Para $\mathrm{s}=1, \ldots, \mathrm{k}$, seja o agrupamento $\mathrm{N}_{\mathrm{s}}$ e o subconjunto $\mathrm{V}_{\mathrm{s}}$ associado;

1.1 Para cada $\mathrm{h} \in \mathrm{V}_{\mathrm{s}}$, considere $\mathrm{w}_{\mathrm{h}} \in\left\{\left(\mathrm{L}_{\min }-\mathrm{S}_{2}\right),\left(\mathrm{L}_{\min }-\mathrm{S}_{2}\right)+1, \ldots,\left(\mathrm{L}_{\max }-\mathrm{S}_{2}\right)\right\}$ a capacidade do compartimento viável de índice $\mathrm{h}$, e calcule um Limitante Superior $\overline{\mathrm{v}}_{\mathrm{h}}$ para a utilidade $\mathrm{v}_{\mathrm{h}}$ do compartimento (por exemplo, o Limitante de Martello-Toth);

2. Seja $V=V_{1} \cup \cdots \cup V_{k}$ e resolva a seguinte mochila:

$$
\begin{aligned}
& \text { maximizar } \sum_{\mathrm{h} \in \mathrm{V}}\left(\overline{\mathrm{v}}_{\mathrm{h}}-\mathrm{c}_{\mathrm{h}}\right) \mathrm{y}_{\mathrm{h}} \\
& \text { sujeito a: } \\
& \qquad \sum_{\mathrm{h} \in \mathrm{V}} \mathrm{w}_{\mathrm{h}} \mathrm{y}_{\mathrm{h}} \leq \mathrm{L}-\mathrm{S}_{1} \\
& \mathrm{y}_{\mathrm{h}} \geq 0 \text { e inteiro, } \mathrm{h} \in \mathrm{V}
\end{aligned}
$$

Na tabela 1 resumimos os resultados que obtivemos para 900 exemplos de mochilas compartimentadas, geradas aleatoriamente. A capacidade de todas as mochilas é 1200, as larguras $\ell_{\mathrm{i}}$ e as utilidades $\mathrm{u}_{\mathrm{i}}$ foram geradas aleatoriamente, respeitando as condições reais do PCBA. O custo por utilizar um compartimento foi considerado como nulo.

Os algoritmos foram implementados em Delphi e executados num pentium II, $450 \mathrm{Mhz}$ e com $160 \mathrm{Mb}$ de RAM. Os exemplos foram agrupados em 3 grandes categorias, segundo o número de compartimentos, e cada uma foi dividida em duas outras, segundo o número de agrupamentos. A menor compartimentação é composta por 30 compartimentos e 3 agrupamentos com 5 itens em cada. A maior compartimentação é formada por 6000 compartimentos e 20 agrupamentos com 100 itens em cada. As colunas da tabela 1 são:

$\boldsymbol{L}_{\min }^{s}$ e $\boldsymbol{L}_{\max }^{s}$ : respectivamente, o limite mínimo e máximo das capacidades dos compartimentos;

Agrup : número de agrupamentos da compartimentação;

Itens : número de itens num agrupamento da compartimentação;

Igualdade : percentual de exemplos em que as soluções do COMPEX e COMPMT foram iguais;

Tempo : tempo médio de execução de um exemplo;

Perda : percentual de perda (espaço ocioso) na mochila;

Dif : percentual de diferença entre o valor da solução do COMPEX e do COMPMT calculado pela expressão 100. $\left|\left(1-\frac{\mathrm{Val}_{\mathrm{COMPMT}}}{\mathrm{Val}_{\mathrm{COMPEX}}}\right)\right|$, onde $\mathrm{Val}_{\mathrm{COMPEX}}$ é o valor do objetivo obtido pela solução do COMPEX, e $\mathrm{Val}_{\text {COMPMT }}$ o valor do objetivo obtido pela solução do COMPMT. 
Tabela 1 - Resultados numéricos de 900 exemplos do PMC

\begin{tabular}{|c|c|c|c|c|c|c|c|c|c|}
\hline \multicolumn{6}{|c|}{$c=1200$} & \multicolumn{2}{|c|}{ COMPEX } & \multicolumn{2}{|c|}{ COMPMT } \\
\hline$L_{\min }^{s}$ & $L_{\max }^{s}$ & Agrup & Itens & Dif & Igualdade & Tempo & Perda & Tempo & Perda \\
\hline \multirow{6}{*}{31} & \multirow{6}{*}{40} & \multirow{3}{*}{3} & 5 & $0,54 \%$ & $74,00 \%$ & $0,110 \mathrm{seg}$ & $0,23 \%$ & $0,050 \mathrm{seg}$ & $0,41 \%$ \\
\hline & & & 40 & $0,29 \%$ & $22,00 \%$ & $0,110 \mathrm{seg}$ & --- & $0,050 \mathrm{seg}$ & $0,20 \%$ \\
\hline & & & 100 & $0,17 \%$ & $10,00 \%$ & $0,112 \mathrm{seg}$ & --- & $0,055 \mathrm{seg}$ & --- \\
\hline & & \multirow{3}{*}{20} & 5 & $0,19 \%$ & $68,00 \%$ & $0,330 \mathrm{seg}$ & --- & $0,083 \mathrm{seg}$ & $0,11 \%$ \\
\hline & & & 40 & $0,05 \%$ & $14,00 \%$ & $0,375 \mathrm{seg}$ & --- & $0,095 \mathrm{seg}$ & $0,06 \%$ \\
\hline & & & 100 & $0,01 \%$ & $8,00 \%$ & $0,380 \mathrm{seg}$ & --- & $0,110 \mathrm{seg}$ & $0,01 \%$ \\
\hline \multirow{6}{*}{51} & \multirow{6}{*}{100} & \multirow{3}{*}{3} & 5 & $0,29 \%$ & $76,00 \%$ & $0,110 \mathrm{seg}$ & $0,26 \%$ & $0,045 \mathrm{seg}$ & $0,48 \%$ \\
\hline & & & 40 & $0,15 \%$ & $52,00 \%$ & $0,126 \mathrm{seg}$ & --- & $0,069 \mathrm{seg}$ & $0,09 \%$ \\
\hline & & & 100 & $0,07 \%$ & $24,00 \%$ & $0,129 \mathrm{seg}$ & --- & $0,071 \mathrm{seg}$ & $0,03 \%$ \\
\hline & & \multirow{3}{*}{20} & 5 & $0,29 \%$ & $62,00 \%$ & $0,470 \mathrm{seg}$ & $0,03 \%$ & $0,170 \mathrm{seg}$ & $0,18 \%$ \\
\hline & & & 40 & $0,17 \%$ & $34,00 \%$ & $0,625 \mathrm{seg}$ & --- & $0,330 \mathrm{seg}$ & $0,05 \%$ \\
\hline & & & 100 & $0,02 \%$ & $24,00 \%$ & $0,741 \mathrm{seg}$ & --- & 0,379 seg & $0,02 \%$ \\
\hline \multirow{6}{*}{151} & \multirow{6}{*}{450} & \multirow{3}{*}{3} & 5 & $1,10 \%$ & $60,00 \%$ & $0,173 \mathrm{seg}$ & $0,84 \%$ & $0,088 \mathrm{seg}$ & $1,68 \%$ \\
\hline & & & 40 & $0,38 \%$ & $22,00 \%$ & $0,361 \mathrm{seg}$ & $0,11 \%$ & $0,197 \mathrm{seg}$ & $0,30 \%$ \\
\hline & & & 100 & $0,33 \%$ & $20,00 \%$ & $0,380 \mathrm{seg}$ & $0,02 \%$ & $0,218 \mathrm{seg}$ & $0,15 \%$ \\
\hline & & \multirow{3}{*}{20} & 5 & $0,90 \%$ & $58,00 \%$ & $1,050 \mathrm{seg}$ & $0,17 \%$ & $0,550 \mathrm{seg}$ & $0,64 \%$ \\
\hline & & & 40 & $0,40 \%$ & $16,00 \%$ & $4,547 \mathrm{seg}$ & $0,01 \%$ & $3,507 \mathrm{seg}$ & $0,22 \%$ \\
\hline & & & 100 & $0,29 \%$ & $18,00 \%$ & $6,150 \mathrm{seg}$ & --- & $3,570 \mathrm{seg}$ & $0,12 \%$ \\
\hline
\end{tabular}

Os resultados obtidos indicam que o procedimento COMPMT é competitivo em relação ao COMPEX para problemas em que o número de compartimentos é alto, porém, ressaltamos que o desempenho deste procedimento pode não ser satisfatório para alguma classe de problemas, visto que, trata-se de uma heurística. Nos problemas maiores, o COMPMT obteve menos soluções ótimas, entretanto, a diferença dos objetivos não se mostrou significativa.

\section{Arredondamento de uma Solução do PCBA e Fracionamento do Estoque}

O procedimento consiste em resolver o modelo (1.1-1.4) relaxando a condição de integralidade da variável $\mathrm{x}_{\mathrm{j}}^{\mathrm{r}}$. A seguir, truncamos (arredondamos para baixo) a solução obtida. Um problema residual é formulado a partir da demanda remanescente e resolvido, de modo que, este processo é repetido até que o arredondamento forneça uma solução residual nula. Ao final, é bem possível que uma pequena demanda ainda deva ser atendida, isto é feito por meio de uma heurística que gera padrões restritos, que também é utilizada nos problemas residuais.

Seja $x^{r}=\left(x_{1}^{r}, \ldots, x_{p_{r}}^{r}\right)^{T}, r=1, \ldots, m$. Observe que $A_{1} x^{1}+A_{2} x^{2}+\cdots+A_{m} x^{m}=d$, e que 1. $x^{r} \leq e^{r}$, onde $A_{r}=\left(\alpha_{i j}^{r}\right)_{n \times p_{r}}$ e $1=(1, \ldots, 1)_{p_{r}}, r=1, \ldots, m$. 
Sejam $x_{0}^{\mathrm{r}}$ a solução contínua do problema $(1.1-1.4)$ e $\left\lfloor\mathrm{x}_{0}^{\mathrm{r}}\right\rfloor$ o arredondamento para baixo desta solução (maior inteiro menor ou igual a $\mathrm{x}_{0}^{\mathrm{r}}$ ), $\mathrm{r}=1, \ldots, \mathrm{m}$. Considere $\mathrm{d}_{0}=\mathrm{d} \quad \mathrm{a}$ demanda inicial e $\mathrm{e}_{0}^{\mathrm{r}}=\mathrm{e}^{\mathrm{r}}, \mathrm{r}=1, \ldots, \mathrm{m}$, a disponibilidade inicial do estoque. Para $q=1, \ldots, Q$, os Problemas Residuais, com soluções contínuas $x_{q}^{r}$, são definidos como:

minimizar $c^{1} x^{1}+\cdots+c^{m} x^{m}$

sujeito a:

$$
\begin{aligned}
& \mathrm{A}_{1} \mathrm{x}^{1}+\cdots+\mathrm{A}_{\mathrm{m}} \mathrm{x}^{\mathrm{m}}=\mathrm{d}_{\mathrm{q}}, \text { onde } \\
& \mathrm{d}_{\mathrm{q}}=\mathrm{d}_{\mathrm{q}-1}-\left(\mathrm{A}_{1}\left\lfloor\mathrm{x}_{\mathrm{q}-1}^{1}\right\rfloor+\cdots+\mathrm{A}_{\mathrm{m}}\left\lfloor\mathrm{x}_{\mathrm{q}-1}^{\mathrm{m}}\right\rfloor\right) \\
& \text { 1. } \mathrm{x}^{\mathrm{r}} \leq \mathrm{e}_{\mathrm{q}-1}^{\mathrm{r}}-\left\|\left\lfloor\mathrm{x}_{\mathrm{q}-1}^{\mathrm{r}}\right\rfloor\right\|_{1}, \mathrm{r}=1, \ldots, \mathrm{m} \\
& \mathrm{x}^{\mathrm{r}} \geq 0, \mathrm{r}=1, \ldots, \mathrm{m}
\end{aligned}
$$

No objetivo do modelo (4.1-4.4) $\quad \mathrm{c}^{\mathrm{r}}=\left(\mathrm{c}_{1}^{\mathrm{r}}, \ldots, \mathrm{c}_{\mathrm{p}_{\mathrm{r}}}^{\mathrm{r}}\right) \quad$ e $\quad \mathrm{c}_{\mathrm{j}}^{\mathrm{r}}=\mathrm{c}_{\mathrm{aço}}^{\mathrm{r}} \mathrm{T}_{\mathrm{j}}^{\mathrm{r}}+\sum_{\mathrm{h}=1}^{\mathrm{H}_{\mathrm{jr}}}\left(\mathrm{c}_{\mathrm{hj}}^{\mathrm{r}} \mathrm{y}_{\mathrm{hj}}^{\mathrm{r}}\right)$, $\mathrm{j}=1, \ldots, \mathrm{p}_{\mathrm{r}}, \mathrm{r}=1, \ldots, \mathrm{m}$. Na inequação $4.3\left\|\left\lfloor\mathrm{x}_{\mathrm{q}-1}^{\mathrm{r}}\right\rfloor\right\|_{1}$ é a norma 1 do vetor $\left\lfloor\mathrm{x}_{\mathrm{q}-1}^{\mathrm{r}}\right\rfloor$. O total Q de Problemas Residuais a serem resolvidos é determinado quando $\left\|\left\lfloor\mathrm{x}_{\mathrm{Q}-1}^{\mathrm{r}}\right\rfloor\right\|_{1}=0$.

Ressaltamos que a medida que os Problemas Residuais são resolvidos as demandas $d_{i}$ das fitas vão diminuindo, por conseqüência, os padrões compartimentados destes problemas devem ser restritos. O modelo do PMC restrito que adotamos é o seguinte:

\section{Modelo do Gerador de Padrões Compartimentados Restritos do PCBA}

$\operatorname{maximizar} \sum_{h \in V_{1}}\left(\left(\sum_{i \in N_{1}} u_{i} a_{i h}\right)-c_{h}\right) y_{h}+\cdots+\sum_{h \in V_{k}}\left(\left(\sum_{i \in N_{k}} u_{i} a_{i h}\right)-c_{h}\right) y_{h}$

sujeito a:

$$
\begin{aligned}
& \sum_{h \in V_{1}}\left(S_{2}+\sum_{i \in N_{1}} \ell_{i} a_{i h}\right) y_{h}+\cdots+\sum_{h \in V_{k}}\left(S_{2}+\sum_{i \in N_{k}} \ell_{i} a_{i h}\right) y_{h} \leq L-S_{1} \\
& L_{\text {min }}-S_{2} \leq \sum_{i \in N_{s}} \ell_{i} a_{i h} \leq L_{\max }-S_{2}, h \in V_{s}, s=1, \ldots, k \\
& \sum_{h \in V_{l}} a_{i h} y_{h}+\ldots+\sum_{h \in V_{k}} a_{i h} y_{h} \leq d_{i}, i \in N \\
& y_{h} \in\{0,1\}, a_{i h} \geq 0 \text { e inteiro, } \\
& i \in N=N_{1} \cup \cdots \cup N_{k}, h \in V=V_{1} \cup \cdots \cup V_{k}
\end{aligned}
$$


No modelo anterior as restrições adicionais são as (5.4) que limitam o total de itens a serem compartimentados na mochila, conforme a demanda de cada fita.

O procedimento COMPEX pode ser adaptado para gerar padrões compartimentados restritos. Isto é feito substituindo as mochilas do passo 1.1 por mochilas canalizadas, cuja restrição de canalização sob as variáveis é baseada nas restrições (5.4). Por fim, a mochila do passo 2 do COMPEX deve ser substituída por uma mochila de variáveis binárias. Com estas modificações temos um procedimento heurístico que denominamos COMPREST para obter uma solução viável do PMC restrito. Se neste novo procedimento, substituirmos o cálculo das mochilas canalizadas pelo simples cálculo de Limitantes Superiores, teremos um novo procedimento heurístico que denominamos COMPRESTMT.

Após a resolução de todos os Problemas Residuais, ainda poderá existir uma demanda residual que é atendida por uma heurística de repetição exaustiva (Hinxman, 1980) com padrões compartimentados restritos.

Em visita a uma empresa do setor descobrimos que as bobinas em estoque poderiam ser fracionadas em duas outras bobinas, cujos "pesos" são exatamente a metade do "peso" da bobina original. Por exemplo, uma bobina do estoque que tenha $12.000 \mathrm{Kg}$ poderia ser fracionada em duas de $6.000 \mathrm{Kg}$.

Assim, o arredondamento das soluções contínuas dos Programas de Programação Linear, usados no Corte de Bobinas de Aço sujeitas a Laminação, pode ser feito por meio da seguinte função:

$$
\llbracket \cdot \rrbracket: \mathbb{R}_{+} \rightarrow \mathbb{N} \cup\left\{n+\frac{1}{2} \mid n \in \mathbb{N}\right\}, \text { onde } \llbracket x \rrbracket=\left\{\begin{array}{l}
\lfloor x\rfloor+0.5, \text { se } x-\lfloor x\rfloor \geq 0.5 \\
\lfloor x\rfloor, \text { se } x-\lfloor x\rfloor<0.5
\end{array}\right.
$$

Observe que ao utilizarmos a função de arredondamento $\llbracket \cdot \rrbracket$, surgirão em estoque bobinas fracionadas, de modo que, ao arredondarmos uma solução torna-se necessário identificarmos se o tipo de bobina em questão já sofreu fracionamento. Caso a bobina em questão tenha sofrido o fracionamento ela não poderá ser novamente fracionada, e o arredondamento deverá ser feito pela função $\lfloor x\rfloor$ (maior inteiro menor ou igual a x).

\section{Resultados Computacionais da aplicação do PMC no PCBA}

As implementações foram feitas em Delphi e executadas num pentium II, $450 \mathrm{Mhz}$ com $160 \mathrm{Mb}$ de RAM. Para construir um padrão compartimentado irrestrito implementamos os procedimentos COMPEX e COMPMT (Hoto, 2001) e (Hoto et al., 2002) que resolvem o Problema da Mochila Compartimentada Irrestrito. Para os padrões compartimentados restritos implementamos os procedimentos COMPREST e COMPRESTMT (Hoto, 2001). Cada exemplo do PCBA foi resolvido de quatro maneiras distintas, obtidas pelas seguintes combinações:

- combinação 1 COMPMT para padrões irrestritos COMPRESTMT para padrões restritos

- combinação 3 COMPEX para padrões irrestritos COMPRESTMT para padrões restritos

\section{- combinação 2} COMPMT para padrões irrestritos COMPREST para padrões restritos

- combinação 4 COMPEX para padrões irrestritos COMPREST para padrões restritos 
Na tabela 2 apresentamos os resultados numéricos que obtivemos na resolução de 21 exemplos gerados aleatoriamente, cujos dados foram baseados nas condições práticas do problema. As larguras foram geradas com valores distribuídos entre $55 \mathrm{~mm}$ e $250 \mathrm{~mm}$.

Tabela 2 - Resultados numéricos de exemplos aleatórios do PCBA

\begin{tabular}{|c|c|c|c|c|c|c|c|c|c|c|c|c|c|c|}
\hline \multicolumn{3}{|c|}{$\begin{array}{l}\text { Dados do } \\
\text { Exemplo }\end{array}$} & \multicolumn{3}{|c|}{$\begin{array}{c}\text { Combinação } 1 \\
\text { COMPMT } \\
\text { COMPRESTMT }\end{array}$} & \multicolumn{3}{|c|}{$\begin{array}{c}\text { Combinação } 2 \\
\text { COMPMT } \\
\text { COMPREST }\end{array}$} & \multicolumn{3}{|c|}{$\begin{array}{c}\text { Combinação } 3 \\
\text { COMPEX } \\
\text { COMPRESTMT }\end{array}$} & \multicolumn{3}{|c|}{$\begin{array}{c}\text { Combinação } 4 \\
\text { COMPEX } \\
\text { COMPREST }\end{array}$} \\
\hline grup & $\mathrm{mp}$ & Itens & Tempo & Perda & Bobint & Tempo & Perda & Bobint & & Perda & Bobint & Tempo & Perda & Bobir \\
\hline & \multirow{5}{*}{1515} & 32 & 10 & - & 773 & 01:39:410 & --- & 768 & $57: 210$ & 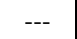 & 581 & $5: 47: 890$ & -1 & 579 \\
\hline & & 32 & $: 41: 630$ & $0,70 \%$ & 748 & 03:11:800 & $0,70 \%$ & 738 & 02:14:290 & --- & 562 & $4: 25: 400$ & $0,01 \%$ & 545 \\
\hline & & 27 & $0: 27: 580$ & $0,51 \%$ & 711 & 01:08:660 & $0,51 \%$ & 704 & $01: 33: 430$ & --- & 506 & $04: 39: 410$ & --- & 511 \\
\hline & & 32 & 57:290 & $0,58 \%$ & 784 & 06:880 & $0,58 \%$ & 88 & 32:000 & -- & 696 & 25.010 & --- & 686 \\
\hline & & 35 & $: 42: 240$ & $1,17 \%$ & 807 & 02:07:420 & $1,19 \%$ & 791 & $01: 48: 480$ & -- & 601 & $05: 43: 170$ & 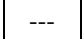 & 588 \\
\hline \multirow{5}{*}{10} & \multirow{5}{*}{3030} & 62 & $: 40: 350$ & $0,04 \%$ & 472 & $28: 180$ & --- & +70 & $05: 26: 260$ & --- & 268 & 10.34 .03 & 0,02 & 1275 \\
\hline & & 62 & $02: 05: 230$ & --- & 1318 & 10:02:420 & $0,01 \%$ & 1330 & 03:50:080 & --- & 1296 & 19:52:870 & $0,05 \%$ & 1275 \\
\hline & & 52 & $01: 32: 270$ & $0,08 \%$ & 1233 & $10: 21: 700$ & --- & 1231 & 05:08:900 & --- & 1198 & 10:38:830 & 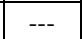 & 1209 \\
\hline & & 60 & $: 30: 440$ & $0,38 \%$ & 1492 & $2: 41: 260$ & $0,39 \%$ & 1481 & $04: 21: 340$ & --- & 1162 & $10.15: 4 / 0$ & --- & 173 \\
\hline & & 57 & $: 44: 000$ & $0,99 \%$ & 1347 & 05:57:340 & $0,99 \%$ & 1380 & $05: 25: 650$ & -- & 1111 & $16: 15: 750$ & $0,03 \%$ & 1084 \\
\hline \multirow{5}{*}{15} & \multirow{5}{*}{4545} & 01 & 00 & 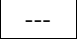 & 77 & 30 & 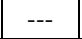 & 86 & 770 & 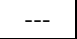 & 341 & 4:10:960 & $0,01 \%$ & 1347 \\
\hline & & 85 & 03:10:980 & --- & 2014 & $14: 43: 470$ & --- & 2007 & $13: 27: 240$ & --- & 1556 & 29:43:870 & -- & 1583 \\
\hline & & 82 & $02: 37: 800$ & $0,67 \%$ & 1948 & $15: 24: 400$ & $0,67 \%$ & 1957 & $07: 49: 880$ & --- & 1545 & $09: 26: 280$ & --- & 1538 \\
\hline & & 82 & 380 & $0,02 \%$ & 2146 & 0 & --- & 2126 & 280 & -- & 1634 & 720 & $0,01 \%$ & 1648 \\
\hline & & 75 & $02: 24: 340$ & $2,77 \%$ & 1676 & $17: 29: 410$ & $0,77 \%$ & 1663 & 08:53:770 & --- & 1442 & 14:40:180 & --- & 1431 \\
\hline \multirow{5}{*}{20} & \multirow{5}{*}{6060} & 95 & $4: 18: 530$ & $1,04 \%$ & 2144 & 11:41:840 & $1,07 \%$ & 2146 & 21:16:080 & $0,01 \%$ & 1983 & $33: 59: 110$ & --- & 1989 \\
\hline & & 110 & $06: 54: 200$ & $1,24 \%$ & 2495 & 23:14:950 & $1,25 \%$ & 2501 & $30: 23: 470$ & --- & 1987 & $38: 21: 380$ & --- & 1974 \\
\hline & & 120 & $07: 23: 250$ & $4,12 \%$ & 2675 & $41: 31: 640$ & $4,18 \%$ & 2674 & $26: 38: 990$ & --- & 2568 & $49: 17: 680$ & --- & 2581 \\
\hline & & 102 & $03: 27: 350$ & $1,22 \%$ & 2286 & $24: 45: 400$ & $1,22 \%$ & 2312 & 13:04:830 & --- & 2175 & 29:05:480 & --- & 2216 \\
\hline & & 100 & 8:04:990 & $0,01 \%$ & 2482 & $14: 24: 960$ & --- & 2460 & $12: 59: 390$ & --- & 2029 & $30: 34: 180$ & --- & 2071 \\
\hline 30 & 90 & 142 & $17: 15: 180$ & --- & 3618 & $38: 28: 740$ & --- & 3602 & $32: 03: 770$ & 0,019 & 3200 & 61:07:010 & --- & 3218 \\
\hline
\end{tabular}

Como estoque utilizamos três tipos de bobinas: com largura de $900 \mathrm{~mm}$, de $1100 \mathrm{~mm}$ e de $1200 \mathrm{~mm}$, todas elas com $12.000 \mathrm{Kg}$. A capacidade do laminador está compreendida entre os valores $154 \mathrm{~mm}$ e $456 \mathrm{~mm}$. O custo de bobinas intermediárias sujeitas à laminação foi considerado $50 \%$ maior que o de bobinas intermediárias isentas deste processo.

Nas colunas Agrup, Comp e Iens estão registrados respectivamente o número de agrupamentos, o número total de compartimentos e o número de itens de cada exemplo. Cada exemplo foi examinado segundo as quatro combinações possíveis, de forma que, foi medido o tempo de execução em minutos, segundos e milisegundos (coluna Tempo), a perda de material (coluna Perda) e o número de bobinas intermediárias no processo (coluna Bobint).

Observe pelo gráfico da figura 5 que as combinações 1 e 2 são praticamente equivalentes quanto ao número de bobinas intermediárias, ocorrendo o mesmo com as combinações 3 e 4 . 


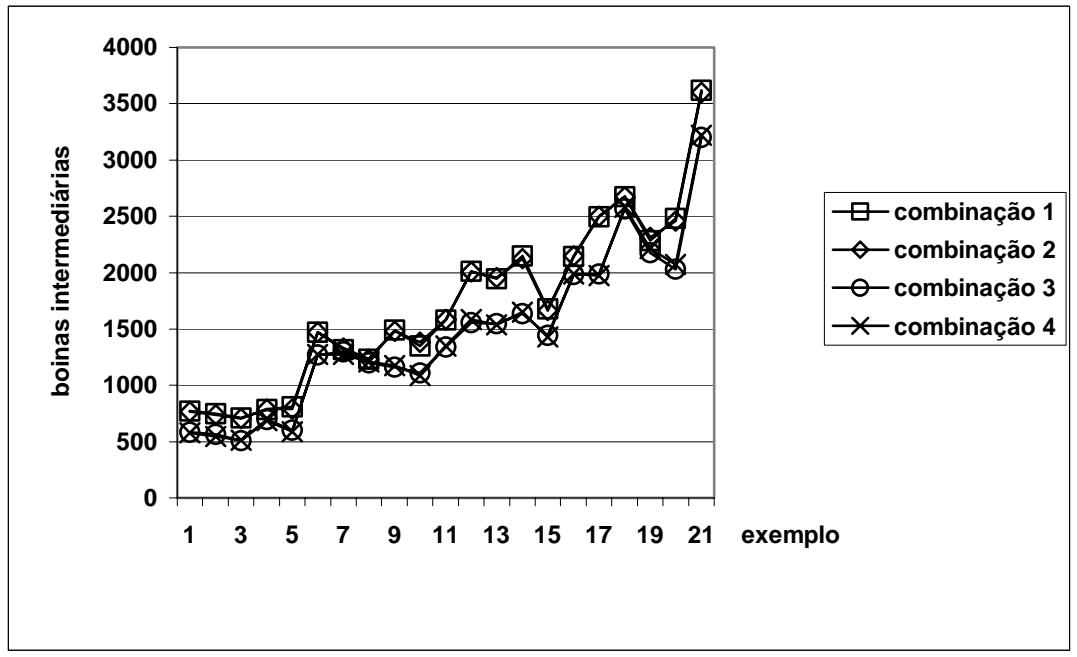

Figura 5 - Gráfico da evolução do número de bobinas intermediárias para cada combinação

No gráfico da figura 6 podemos observar que a combinação que requer menor tempo de execução na maioria dos exemplos é a combinação 1 , porém, o número de bobinas intermediárias é bem superior se comparados com os obtidos pela combinação 4, que requer um tempo de execução mais elevado.

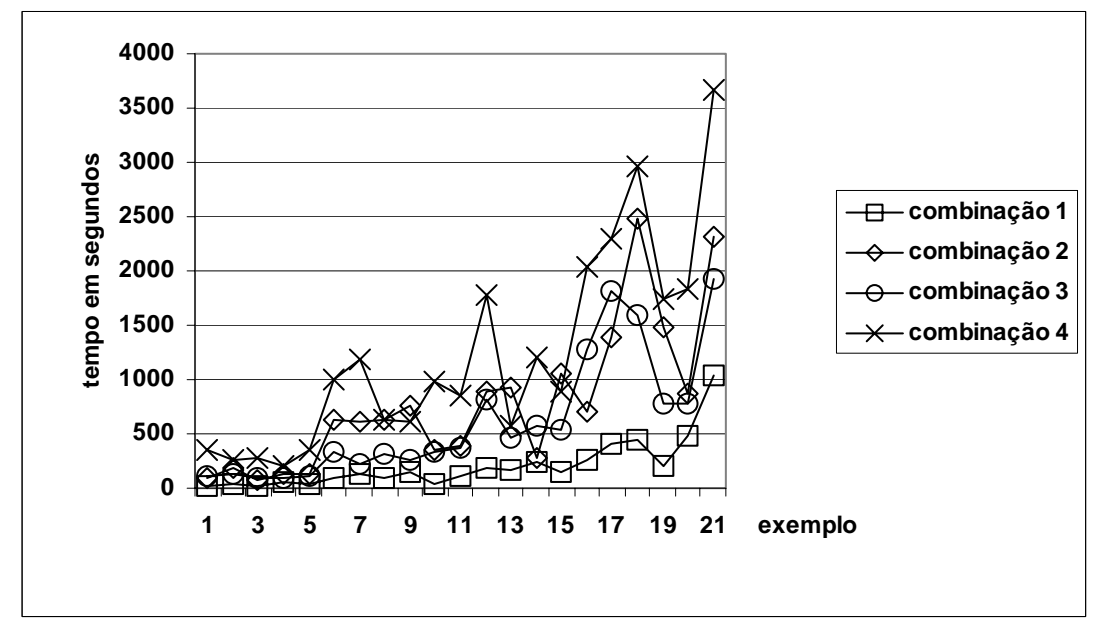

Figura 6 - Gráfico da evolução do tempo de execução para cada combinação

Para finalizar, apresentamos os resultados obtidos para um conjunto de 20 itens extraídos de dados reais. Os agrupamentos dos itens são: $N_{1}=\{1,2,3\}, \quad N_{2}=\{4\}, \quad N_{3}=\{5\}$, $\mathrm{N}_{4}=\{6,7,8\}, \mathrm{N}_{5}=\{9,10,11,12\}, \mathrm{N}_{6}=\{13,14,15,16,17\}$ e $\mathrm{N}_{7}=\{18,19,20\}$. Na tabela 3 estão os resumidos os dados do problema e na tabela 4 os resultados obtidos. 
Tabela 3 - Um exemplo do PCBA extraído de dados reais

\begin{tabular}{|c|c|c|c|c|c|}
\hline Fitas & Largura & $\begin{array}{c}\text { Espessura } \\
\text { Inicial }\end{array}$ & $\begin{array}{c}\text { Espessura } \\
\text { Final }\end{array}$ & Tipos de Aço & Demandas \\
\hline tipo 1 & $200 \mathrm{~mm}$ & $2,00 \mathrm{~mm}$ & $1,10 \mathrm{~mm}$ & $S A E 1008$ & $50.000 \mathrm{Kg}$ \\
\hline tipo 2 & $230 \mathrm{~mm}$ & $2,00 \mathrm{~mm}$ & $1,10 \mathrm{~mm}$ & $S A E 1008$ ou $S A E 1010$ & $55.000 \mathrm{Kg}$ \\
\hline tipo 3 & $90 \mathrm{~mm}$ & $2,00 \mathrm{~mm}$ & $1,10 \mathrm{~mm}$ & $S A E 1008$ & $60.000 \mathrm{Kg}$ \\
\hline tipo 4 & $115 \mathrm{~mm}$ & $2,00 \mathrm{~mm}$ & $1,20 \mathrm{~mm}$ & $S A E 1008$ & $57.500 \mathrm{Kg}$ \\
\hline tipo 5 & $105 \mathrm{~mm}$ & $2,00 \mathrm{~mm}$ & $1,10 \mathrm{~mm}$ & $S A E 1010$ & $63.000 \mathrm{Kg}$ \\
\hline tipo 6 & $105 \mathrm{~mm}$ & $1,50 \mathrm{~mm}$ & $0,90 \mathrm{~mm}$ & $S A E 1010$ ou $S A E 1012$ & $47.000 \mathrm{Kg}$ \\
\hline tipo 7 & $200 \mathrm{~mm}$ & $1,50 \mathrm{~mm}$ & $0,90 \mathrm{~mm}$ & $S A E 1012$ & $71.000 \mathrm{Kg}$ \\
\hline tipo 8 & $120 \mathrm{~mm}$ & $1,50 \mathrm{~mm}$ & $0,90 \mathrm{~mm}$ & $S A E 1010$ ou $S A E 1012$ & $54.000 \mathrm{Kg}$ \\
\hline tipo 9 & $90 \mathrm{~mm}$ & $2,00 \mathrm{~mm}$ & $2,00 \mathrm{~mm}$ & $S A E 1008$ & $53.800 \mathrm{Kg}$ \\
\hline tipo 10 & $70 \mathrm{~mm}$ & $2,00 \mathrm{~mm}$ & $2,00 \mathrm{~mm}$ & $S A E 1008$ & $70.200 \mathrm{Kg}$ \\
\hline tipo 11 & $65 \mathrm{~mm}$ & $2,00 \mathrm{~mm}$ & $2,00 \mathrm{~mm}$ & $S A E 1008$ ou $S A E 1010$ & $61.000 \mathrm{Kg}$ \\
\hline tipo 12 & $105 \mathrm{~mm}$ & $2,00 \mathrm{~mm}$ & $2,00 \mathrm{~mm}$ & $S A E 1008$ & $50.000 \mathrm{Kg}$ \\
\hline tipo 13 & $135 \mathrm{~mm}$ & $1,00 \mathrm{~mm}$ & $1,00 \mathrm{~mm}$ & $S A E 1010$ ou $S A E 1012$ & $55.550 \mathrm{Kg}$ \\
\hline tipo 14 & $205 \mathrm{~mm}$ & $1,00 \mathrm{~mm}$ & $1,00 \mathrm{~mm}$ & $S A E 1010$ & $48.600 \mathrm{Kg}$ \\
\hline tipo 15 & $125 \mathrm{~mm}$ & $1,00 \mathrm{~mm}$ & $1,00 \mathrm{~mm}$ & $S A E 1010$ & $49.000 \mathrm{Kg}$ \\
\hline tipo 16 & $115 \mathrm{~mm}$ & $1,00 \mathrm{~mm}$ & $1,00 \mathrm{~mm}$ & $S A E 1010$ & $52.500 \mathrm{Kg}$ \\
\hline tipo 17 & $180 \mathrm{~mm}$ & $1,00 \mathrm{~mm}$ & $1,00 \mathrm{~mm}$ & $S A E 1010$ & $63.000 \mathrm{Kg}$ \\
\hline tipo 18 & $53 \mathrm{~mm}$ & $1,50 \mathrm{~mm}$ & $1,50 \mathrm{~mm}$ & $S A E 1010$ ou $S A E 1012$ & $60.500 \mathrm{Kg}$ \\
\hline tipo 19 & $105 \mathrm{~mm}$ & $1,50 \mathrm{~mm}$ & $1,50 \mathrm{~mm}$ & $S A E 1012$ & $58.200 \mathrm{Kg}$ \\
\hline tipo 20 & $90 \mathrm{~mm}$ & $1,50 \mathrm{~mm}$ & $1,50 \mathrm{~mm}$ & $S A E 1010$ ou $S A E 1012$ & $54.500 \mathrm{Kg}$ \\
\hline & & & & & \\
\hline
\end{tabular}

Tabela 4 - Solução do exemplo da tabela 3

\begin{tabular}{|c|c|c|c|c|c|c|c|c|c|c|c|c|c|c|}
\hline \multicolumn{3}{|c|}{$\begin{array}{l}\text { Dados do } \\
\text { Exemplo }\end{array}$} & \multicolumn{3}{|c|}{$\begin{array}{c}\text { Combinação } 1 \\
\text { COMPMT } \\
\text { COMPRESTMT }\end{array}$} & \multicolumn{3}{|c|}{$\begin{array}{c}\text { Combinação } 2 \\
\text { COMPMT } \\
\text { COMPREST }\end{array}$} & \multicolumn{3}{|c|}{$\begin{array}{c}\text { Combinação } 3 \\
\text { COMPEX } \\
\text { COMPRESTMT }\end{array}$} & \multicolumn{3}{|c|}{$\begin{array}{c}\text { Combinação } 4 \\
\text { COMPEX } \\
\text { COMPREST }\end{array}$} \\
\hline Agrup & Comp & Itens & Tempo & Perda & Bobint & Tempo & Perda & Bobint & Tempo & Perda & Bobint & Tempo & Perda & Bobint \\
\hline 7 & 2121 & 20 & $0: 11: 320$ & $0,77 \%$ & 341 & $00: 12: 200$ & $0,77 \%$ & 342 & $00: 33: 230$ & $0,25 \%$ & 319 & 00:39:710 & $0,25 \%$ & 325 \\
\hline
\end{tabular}

\section{Conclusões}

Neste artigo abordamos o Problema de Corte de Bobinas de Aço (PCBA), que é um problema de corte unidimensional, cujos padrões devem ser estruturados em compartimentos. Para construir um padrão de corte do PCBA é preciso resolver um especial problema da mochila que denominamos Problema da Mochila Compartimentada (PMC).

Para o PMC irrestrito desenvolvemos dois procedimentos o COMPEX e o COMPMT, que foram descritos e comparados numericamente. No caso do COMPEX mostramos que o procedimento encontra uma solução ótima do PMC, já o COMPMT consiste de uma heurística baseada no cálculo de limitantes superiores. 
Para o PMC restrito desenvolvemos um procedimento heurístico derivado do COMPEX que denominamos COMPREST. A abordagem do cálculo de limitantes superiores no lugar das mochilas canalizadas, foi aplicada no COMPREST, fornecendo um procedimento que denominamos COMPRESTMT.

Utilizamos a Técnica de Geração de Colunas de Gilmore-Gomory para resolver o PCBA e, baseado nos resultados computacionais obtidos, os algoritmos COMPEX (para gerar padrões do caso irrestrito) e COMPRESTMT (para gerar padrões do caso restrito) resultam numa boa estratégia para problema.

\section{Agradecimentos}

Este trabalho teve apoio da FAPESP - Fundação de Amparo à Pesquisa de São Paulo, da CAPES - Coordenação de Aperfeiçoamento de Pessoal de Nível Superior, do Ministério da Educação Brasileira e do CNPq - Conselho Nacional de Desenvolvimento Científico e Tecnológico, do Ministério da Educação Brasileira. Ao Professor Nei Yoshihiro Soma do Instituto Tecnológico de Aeronáutica do Brasil, nossos sinceros agradecimentos. Agradecemos também os revisores do trabalho que indicaram valiosas sugestões.

\section{Referências Bibliográficas}

(1) de Carvalho, J.M.V. Valério (1991). Um problema de corte em duas fases. Tese de Doutoramento, Universidade do Minho, Portugal.

(2) de Carvalho, J.M.V. Valério \& Rodrigues, A.J.G. (1994). A computer based interactive approach to a two-stage cutting-stock problem. INFOR, 32(4), 243-252.

(3) de Carvalho, J.M.V. Valério \& Rodrigues, A.J.G. (1995). An LP-based approach to a two-stage cutting-stock problem. European Journal of Operational Research, 84, 580-589.

(4) Ferreira, J.S.; Neves, M.A. \& Castro, P.F. (1990). A two-phase roll cutting problem. European Journal of Operational Research, 44, 185-196.

(5) Gilmore, P.C. \& Gomory, R.E. (1961). A Linear Programming Approach to the Cutting Stock Problem. Operations Research, 9, 849-859.

(6) Gilmore, P.C. \& Gomory, R.E. (1963). A Linear Programming Approach to the Cutting Stock Problem, part II. Operations Research, 14, 94-120.

(7) Gilmore, P.C. \& Gomory, R.E. (1966). The theory and computation of knapsack functions. Operations Research, 14, 1045-1074.

(8) Haessler, R.W. (1975). Controlling cutting pattern changes in one dimensional trim problems. Operations Research, 23(3), 483-493.

(9) Haessler, R.W. (1979). Solving the two-stage cutting-stock problem. Omega, The International Journal of Management Science, 7(2), 145-151.

(10) Haessler, R.W. (1980). A note on computational modifications to the Gilmore-Gomory cutting stock algorithm. Operations Research, 28(4), 1001-1005. 
(11) Hinxman, A.I. (1980). The trim-loss and assortment problems: a survey. European Journal of Operational Research, 5, 8-18.

(12) Hoto, R. (1996). Otimização no Corte de Peças Unidimensionais com Restrições de Agrupamento. Dissertação de Mestrado, ICMSC-USP, São Carlos, SP, Brasil.

(13) Hoto, R. (2001). O Problema da Mochila Compartimentada aplicado no Corte de Bobinas de Aço. Tese de Doutoramento, COPPE-Sistemas/UFRJ, Rio de Janeiro, RJ, Brasil.

(14) Hoto, R. \& Arenales, M. (1996). Um problema de corte unidimensional com restrições de agrupamento e aplicações industriais. I ON PCE, IME-USP, São Paulo, SP, Brasil.

(15) Hoto, R. \& Arenales, M. (1997). O problema do corte em bobinas de aço. XX CNMAC, Gramado, RS, Brasil.

(16) Hoto, R.; Arenales, M.N. \& Maculan, N. (1999). O Problema da Mochila Compartimentada. Relatório Técnico, Departamento de Matemática, Centro de Ciências Exatas, Universidade Estadual de Londrina, Londrina, PR, Brasil.

(17) Hoto, R.; Maculan, N. \& Arenales, M.N. (1998). O problema do corte em bobinas de aço via geração de colunas. XXX Simpósio Brasileiro de Pesquisa Operacional, Curitiba, PR, Brasil, 267-268.

(18) Hoto, R.; Maculan, N.; Arenales, M.N. \& Marques, F.P. (2002). Um novo procedimento para o cálculo de mochilas compartimentadas. Pesquisa Operacional, Lisboa, Portugal, 22(2), 213-234.

(19) Johnston, R.E. \& Khan, L.R. (1995). Bounds for nested knapsack problems. European Journal of Operational Research, 81, 154-165.

(20) Lin, E. Yu-Hsien (1998). A bibliographical survey on some well-known non-standard knapsack problems. INFOR, 36(4), 274-317.

(21) Marques, F.P. (2000). O Problema da Mochila Compartimentada. Dissertação de Mestrado, ICMC-USP, São Carlos, SP, Brasil.

(22) Marques, F.P. \& Arenales, M.N. (2000). O problema da mochila compartimentada. XXXII Simpósio Brasileiro de Pesquisa Operacional, Viçosa, MG, Brasil, 803-816.

(23) Martello, S. \& Toth, P. (1990). Knapsack Problems: Algorithms and Computer Implementations. John Wiley \& Sons, Chichester.

(24) Pereira, M.A. (1993). Uma abordagem matemática para o problema de corte e laminação de fitas de aço. Dissertação de Mestrado, UNICAMP, Campinas, SP, Brasil.

(25) Pisinger, D. (1995). Algorithms for knapsack problems. Ph.D. Thesis, Department of Computer Science, University of Copenhagen, Copenhagen, Denmark.

(26) Suzuki, H. (1978). A generalized knapsack problem with variable coefficients. Mathematical Programming, 15, 162-176.

(27) Stadtler, H. (1990). A one dimensional cutting-stock problem in the aluminium industry and its solution. European Journal of Operational Research, 44, 209-223.

(28) Soma, N.Y. \& Toth, P. (1999). On the critical item for subset sum problems. Revista Pesquisa Operacional - SOBRAPO, 19(2), 279-283. 
(29) Soma, N.Y.; Yanasse, H.H. \& Maculan, N. (1997). O problema da mochila. O Problema de Corte e Empacotamento e Aplicações Industriais, 2.a Oficina Nacional de PCE, XX CNMAC, Gramado, RS, 24-58.

(30) Soma, N.Y.; Yanasse, H.H.; Zinober, A.S.I. \& Harley, P.J. (1995). A polynomial approximation scheme for the subset sum problem. Discrete Applied Mathematics, 57, 243-253.

(31) Wäscher, G. \& Gau, T. (1996). Heuristics for the integer one dimensional cutting-stock problem: a computacional study. OR Spektrum, 18, 131-144.

(32) Yanasse, H.H. \& Soma, N.Y. (1987). A new enumeration scheme for the knapsack problem. Discrete Applied Mathematics, 18, 235-245.

(33) Yanasse, H.H.; Soma, N.Y. \& Maculan, N. (2000). An algorithm for determining the k-best solutions of one-dimensional knapsack problem. Pesquisa Operacional, 20(1), 117-134. 